\title{
Realization of a Continuously Phase-Locked Few-Cycle Deep-UV/XUV Pump-Probe Beamline with Attosecond Precision for Ultrafast Spectroscopy
}

\author{
Tsendsuren Khurelbaatar ${ }^{1,2} \mathbb{D}$, Alexander Gliserin ${ }^{1,2,3} \mathbb{D}$, Je-Hoi Mun ${ }^{1,2} \mathbb{D}$, Jaeuk Heo ${ }^{1,2}$, Yunman Lee ${ }^{1,2}$ \\ and Dong-Eon Kim 1,2,*
}

Citation: Khurelbaatar, T.; Gliserin A.; Mun, J.-H.; Heo, J.; Lee, Y.; Kim, D.-E. Realization of a Continuously Phase-Locked Few-Cycle Deep-UV / XUV Pump-Probe Beamline with Attosecond Precision for Ultrafast Spectroscopy. Appl. Sci. 2021, 11, 6840. https://doi.org/ 10.3390/app11156840

Academic Editors: Vibhav Bharadwaj and Sanathana Konugolu Venkata Sekar

Received: 30 June 2021

Accepted: 22 July 2021

Published: 25 July 2021

Publisher's Note: MDPI stays neutral with regard to jurisdictional claims in published maps and institutional affiliations.

Copyright: (c) 2021 by the authors. Licensee MDPI, Basel, Switzerland. This article is an open access article distributed under the terms and conditions of the Creative Commons Attribution (CC BY) license (https:/ / creativecommons.org/licenses/by/ $4.0 /)$.
1 Center for Attosecond Science and Technology, Department of Physics, Pohang University of Science and Technology, Pohang, Gyeongbuk 37673, Korea; khtsendsuren@postech.ac.kr (T.K.); alex@mpk.or.kr (A.G.); mun1219@mpk.or.kr (J.-H.M.); showerpurple@postech.ac.kr (J.H.); leeym@mpk.or.kr (Y.L.)

2 Max Planck POSTECH/KOREA Research Initiative, Pohang, Gyeongbuk 37673, Korea

3 Department of Optics and Mechatronics Engineering, College of Nanoscience and Engineering, Pusan National University, 2 Busandaehak-ro 63beon-gil, Busan 46241, Korea

* Correspondence: kimd@postech.ac.kr

\begin{abstract}
Chemical and physical processes in molecules can be controlled through the manipulation of quantum interferences between rotational, vibrational, and electronic degrees of freedom. Most of the past efforts have been focused on the control of nuclear dynamics. Even though electronic coherence and its coupling to nuclear degrees of freedom may profoundly affect the outcome of these processes, electron dynamics have received less attention. Proper investigation of electron dynamics in materials demands ultrafast sources in the visible, ultraviolet (UV), and extreme ultraviolet (XUV) spectral region. For this purpose, a few-cycle deep-UV and XUV beamlines have been constructed for studying ultrafast electron dynamics in molecules. To ensure the required high temporal resolution on the attosecond time scale, vibration isolation from environmental mechanical noise and active stabilization have been implemented to achieve attosecond timing control between pump and probe pulses with excellent stability. This is achieved with an actively phase-stabilized double-layer MachZehnder interferometer system capable of continuous time-delay scans over a range of $200 \mathrm{fs}$ with a root-mean-square timing jitter of only 13 as over a few seconds and $\sim 80$ as of peak-to-peak drift over several hours.
\end{abstract}

Keywords: nonlinear optics; high-order harmonic generation; interferometry; jitter-stabilization; attosecond precision; ultrafast spectroscopy

\section{Introduction}

The control of material processes at the level of electron motion in real time has become one of the significant issues in fundamental science [1-3]. The triggering and complete measurement of valence orbitals of neutral electronic systems, which are the most relevant for chemical functions, has hitherto remained inaccessible. For the control of chemical dynamics in a new paradigm, therefore, we need to understand the dynamics beyond the Born Oppenheimer approximation where the electronic states are not stationary and influential. We need to explore how nonstationary electronic states are coupled to nuclear motion and subsequently understand how the manipulation of the electronic motion can be used to trigger a selective motion of the nuclei, thereby implementing a chemical reaction pathway [4-10]. Ultrafast charge migration and conical intersection dynamics are a few examples among many chemical dynamics [11,12]. Ultrafast migration of a charge driven by electron correlation is manifested when ultrashort ultraviolet (UV) laser pulses create electronic wave packets, triggering pure electron dynamics in an ionized system [13].

To facilitate our understanding and control of chemical dynamics in a new paradigm, an intense few-cycle deep-UV (DUV) beamline combined with ultrashort near-infrared 
(NIR) pulses and extreme ultraviolet (XUV) pulses for time-resolved experiments has been developed. Numerous studies have been conducted using the frequency upconversion method in noble gases to generate few-cycle DUV pulses [14-16] and XUV pulses [17-19].

For investigations of few-cycle dynamics with high time resolution, the timing jitter between the pump and probe pulses should be controlled on the attosecond time scale. Several active stabilization methods have been established and are being used in the case of XUV pulses combined with a NIR beam [20-22]. The majority of them use a collinear scheme in which a continuous wave (CW) laser co-propagates as a reference together with the XUV and/or NIR beams in an interferometry setup. However, when few-cycle DUV pulses are combined with XUV pulses in the beamline, such a collinear scheme with a CW laser is not feasible due to spectrally incompatible dielectric and dichroic optics along the beam paths. To overcome these difficulties, a double-layer interferometry (DLI) system was implemented, providing an upper-layer beam path for the reference CW laser and a lower-layer path for the DUV and XUV beams with their respective specialized optics, while maintaining strong mechanical coupling via custom made Al double-mirror mounts and sharing the same mechanical delay stage. Thus, stabilizing and scanning the optical phase in the reference upper-layer interferometer ensures the same behavior of the lowerlayer interferometer's beam path, since both layers are mechanically locked. Additionally, in conventional setups, the relative phase between the two arms of the interferometer must be relocked at each delay step because the intentional delay change breaks the relative phase relationship between the two arms. This becomes an issue when the data acquisition takes a long time and requires automation.

In our DUV / XUV beamline, the DLI system is capable of continuous delay change while maintaining the relative phase between the two interferometer arms. We demonstrate that the relative phase between the few-cycle DUV and XUV arms can be maintained down to a precision of $\sim 13$ as root-mean-square (RMS) on a few-second time scale with a maximum peak-to-peak phase drift of $\sim 80$ as over $2 \mathrm{~h}$ while continuously scanning the delay for the pump-probe experiment.

\section{Ultrafast Deep-UV and XUV Beamline}

The femtosecond DUV and XUV beamline consists of three chambers: source, delay, and target chamber. The schematic diagram is presented in Figure 1a. The beamline is driven by carrier-envelope-phase (CEP) stabilized sub- 5 fs NIR pulses close to the Fouriertransform limit (flat spectral phase) with a pulse energy of $0.25 \mathrm{~mJ}$ at a $3 \mathrm{kHz}$ repetition rate. The spectral phase and resulting pulse duration was characterized by the dispersion scan method [23]. The driving NIR laser pulses are focused into quasi-static gas cells filled with noble gases using a $500 \mathrm{~mm}$ focal length spherical mirror for collinear third- and high-order harmonic generation (THG and HHG, light-yellow panel in Figure 1a) [24]. The THG gas cell is placed in front of the focus, whereas the HHG gas cell is placed about $3 \mathrm{~mm}$ after the focus. The cell is a $3 \mathrm{~mm}$ inner-diameter ceramic tube and has a $200 \mu \mathrm{m}$ diameter hole for the laser beam. One end of the tube is sealed with epoxy. A ceramic tube is chosen because it is robust against damage from the focused laser pulse. Two motorized three-axis stages are used to independently control the two gas cell positions, allowing for fine optimization of the harmonic yields. The driving NIR spectrum is displayed in Figure $1 \mathrm{~b}$ and the generated DUV and XUV spectra are shown in Figure 1c,d, respectively. With careful optimization of the cell position and gas pressure in consideration of the nonlinear effect during the THG, a DUV pulse with an energy of $\sim 1 \mu \mathrm{J}$ has been obtained. The optimization reveals that the THG yield from an Ar gas is the highest at a pressure of 0.8 bar in the cell. The HHG gas cell is supplied with Ne gas of less than 200 mbar. During the THG and HHG process, all chambers are kept under vacuum, and any residual gas is evacuated with turbo molecular pumps and a backing scroll pump. The corresponding vacuum level of each chamber is indicated in Figure 1a. The DUV spectrum in Figure 1c supports a Fourier-transform limited pulse duration of $2.6 \mathrm{fs}$. Since the driving NIR pulse is near-Fourier-transform-limited, we estimate the resulting DUV pulse to be close to the 
Fourier-transform limit as well, since the group velocity dispersion in a gaseous nonlinear medium is negligible compared to a solid medium. Temporal characterization of the XUV pulses can be done via streaking in the future. Based on previous work with comparable driving pulses and XUV spectra, the XUV pulse duration is expected to be in the order of a few 100 as [25]. The photon flux of the XUV source is $\sim 2.5 \times 10^{7}$ photons/pulse at the target chamber, taking into account the losses from the metallic filters and rhodium mirrors.
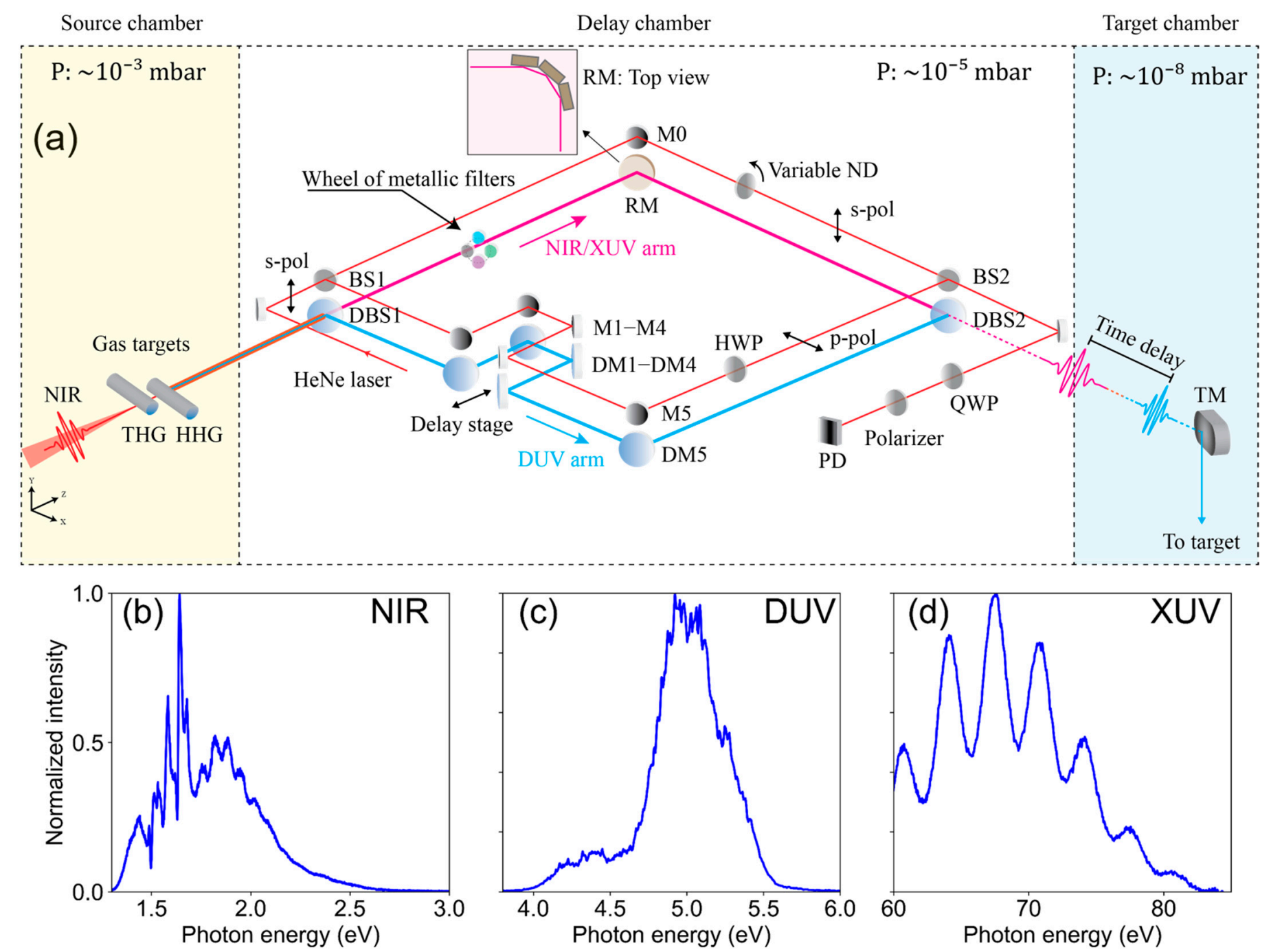

Figure 1. Layout of the experimental setup and the spectra of the generated light sources. (a) Scheme for collinear harmonics generation and the double-layer interferometer method for pump-probe delay and active stabilization. The upper layer contains a reference interferometer for active phase stabilization; the lower layer contains the main beam path for the pump-probe experiment using a DUV and an XUV beam. (b) Driving few-cycle NIR laser spectrum. (c) Measured DUV and (d) XUV spectrum. THG: third-order harmonic generation, HHG: high-order harmonic generation, DBS: dichroic beam separator, BS: HeNe beamsplitter, M: silver mirror, RM: triple rhodium mirror $\left(75^{\circ}\right.$ angle of incidence per segment), DM: dielectric mirror, TM: toroidal mirror, HWP: half-wave plate, QWP: quarter-wave plate, ND: neutral-density filter, PD: photodiode.

After the source chamber, the generated DUV, XUV, and residual fundamental NIR pulses co-propagate into the delay chamber where a home-built double-layer MachZehnder interferometer system provides a variable, actively phase-stabilized pump-probe delay. Inherently different divergences of the fundamental NIR, DUV, and XUV pulses allow us to separate them spatially using a perforated dichroic beam separator (DBS1 in 
Figure 1a, Layertec $\mathrm{GmbH}$, Mellingen, Germany) with a hole of $1.0 \mathrm{~mm}$ diameter, through which the XUV and residual NIR pulses can pass, whereas $>90 \%$ of the DUV beam is reflected off. The XUV beam can pass through the aperture without clipping. Thin metallic filters such as $\mathrm{Al}$ and $\mathrm{Zr}$ (wheel of metallic filters in Figure 1a) allow the XUV pulse to be transmitted while blocking the remaining small portion of the DUV and fundamental NIR pulses. The residual NIR in the DUV arm is effectively suppressed by utilizing the reflectivity properties of the UV separators and dielectric mirrors $(\mathrm{R}>99 \%$ at $270 \mathrm{~nm}$, $\mathrm{R}<10 \%$ at $405-850 \mathrm{~nm}$ ) with a total of nine reflections from the DUV optics, resulting in NIR suppression of more than $99.99 \%$. The thin metal filters in the XUV arm (150-300 nm thickness) suppress the NIR and DUV transmission by at least nine orders of magnitude, allowing for essentially perfect separation of the DUV and XUV beams for pump-probe experiments. The DUV and XUV pulses are then combined again at the second perforated dichroic beam separator (DBS2 in Figure 1a), which has a $1.5 \mathrm{~mm}$ diameter hole. The combined beams are then focused by a toroidal mirror into the interaction chamber where the interaction region is located between the repeller and extractor plates of a velocity map imaging (VMI) spectrometer [26]. In the interaction chamber, the sample under study can be injected either through a supersonic gas jet from the top of the target chamber or through an effusive gas jet which is integrated into the repeller plate of the VMI spectrometer.

\section{Actively Phase-Stabilized Continuous Optical Delay Scan with Attosecond Timing Accuracy}

The uniqueness of the current ultrafast DUV / XUV beamline is the capability of continuous delay scanning with attosecond timing precision. For this purpose, as mentioned above, a double-layer interferometer (DLI) setup with an arm length of $\sim 1.2 \mathrm{~m}$ has been constructed, as shown in the mid-panel of Figure $1 \mathrm{a}$, in which the upper layer serves as a reference interferometer to scan and stabilize the DUV/XUV delay line on the lower layer. To achieve a high degree of phase stabilization, the optical breadboard of the delay line chamber is supported by vibration isolators (VIB320, Newport, Irvine, CA, USA), which greatly suppress the mechanical noise from various sources in the laboratory. In order to minimize the intensity drift of the upper-layer interferometer, which would cause a phase drift of the DUV / XUV delay, an intensity-stabilized HeNe laser is used to provide the phase reference in the upper layer. As shown in Figure 2a, custom-designed Al mirror mounts have been fabricated for the DLI setup to mechanically link the lower- and upper-layer interferometers. Since most mechanical drifts and vibrations in both layers are correlated with this design, the phase locking in the upper-layer interferometer automatically ensures the phase locking in the lower layer. Half-inch standard silver mirrors are used in the upper layer for the HeNe laser, whereas 1 inch dielectric mirrors and perforated dichroic beam separators are installed in the lower layer tailored for the DUV and XUV/IR pulses in the respective beam paths (see Figure 1a).

For continuous scanning of the delay over a long range while actively stabilizing the phase in the DLI setup, we utilize the accumulated Pancharatnam's phase method [27] in the reference upper-layer interferometer. Figure $2 b$ illustrates the active stabilization scheme in which an intensity-stabilized, linearly polarized (s-pol) HeNe laser beam passes through the upper-layer interferometer. After the first beamsplitter (BS1), the polarization in one of the arms is rotated by 90 degrees with respect to the other arm using a half-wave plate (HWP). The cross-polarized beams are then recombined at the second beamsplitter (BS2) and pass through a quarter-wave plate (QWP), which converts these crossed linear polarizations into circular polarizations of opposite helicity. The superposition of the two orthogonal circular polarizations results again in a linearly polarized beam whose polarization angle now only depends on the relative optical phase between the two circular polarization components. Effectively, this method converts a change in optical phase (or delay) into a change of the linear polarization angle, while in a simple interferometer an optical phase change results in an intensity change due to constructive or destructive interference. The advantage of this method is that the rotation of the polarization angle is directional and continuous with the optical delay, while the intensity variation due 
to interference in a normal interferometer changes direction after every half-cycle. This ambiguity of the interference amplitude as a function optical delay limits the scan range of a typical phase-locked interferometer to a half-cycle of the reference laser's wavelength. In contrast, using the Pancharatnam's phase method eliminates this ambiguity, since here a change of the optical delay in one direction results in a directional change of the polarization angle. Continuous tracking of the absolute angle (including the number of turns) allows for a unique mapping between optical delay and polarization angle over an arbitrary range. By analyzing the polarization angle with a polarizer (POL) and detecting the resulting HeNe laser intensity with a photodiode (PD), we can lock the optical delay by keeping the photodiode signal (and thus the polarization angle) at a certain value. This is achieved by using an analog proportional-integral-derivative (PID) controller (SIM960, Stanford Research Systems, Sunnyvale, USA) together with a piezoelectric transducer for the optical delay stage in a feedback loop with the set point set to roughly the middle of the range between the minimum and maximum photodiode signal after the polarizer as a function of optical delay. Delay scanning can now easily be achieved by slowly rotating the polarizer while maintaining the PID lock. Since the polarization change is continuous with the optical delay, the scan range is only limited by the reference laser's coherence length and the mechanical constraints of the optical delay stage. Turning the polarizer results in a unique corkscrew-like relationship between the absolute angle of the polarizer and the optical path difference between the interferometer arms as long as the PID lock is never lost. A full $(2 \pi)$ turn of the polarizer corresponds to an optical phase change of $4 \pi$, or two wavelengths of the reference laser.
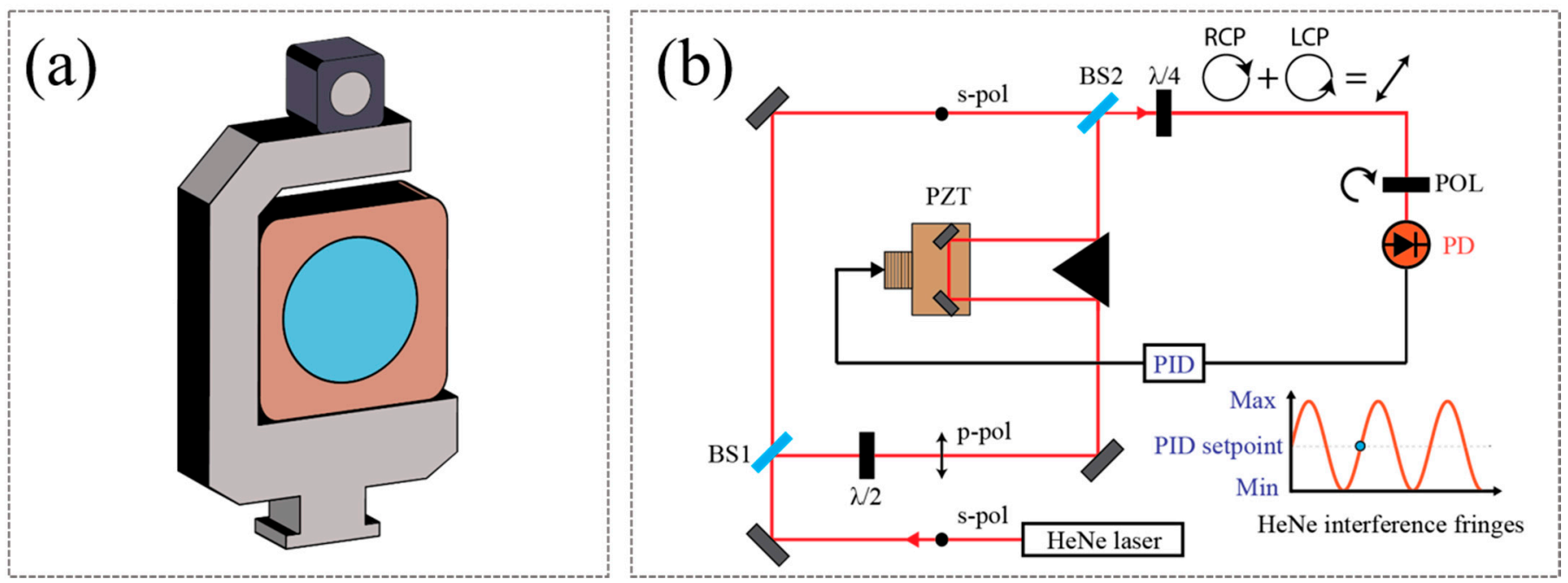

Figure 2. (a) Custom-designed coupled Al mirror mount for the double-layer interferometer setup. (b) Actively-stabilized continuous delay-scan scheme based on Pancharatnam's phase [24] in the upper-layer (reference) interferometer. BS: beamsplitter, PZT: piezoelectric transducer stage, PD: photodiode, POL: polarizer, PID: proportional-integral-derivative controller, RCP: right-handed circular polarization, LCP: left-handed circular polarization.

To validate the performance of the DLI, for simplicity, the dielectric/dichroic mirrors in the lower layer have been replaced with Ag mirrors. The intensity-stabilized $\mathrm{HeNe}$ laser is now split in two parts. One part is used in the upper layer with the PID feedback loop to stabilize and scan the reference interferometer, referred to as in-loop (IL), while the second part is used for lower layer (out-of-loop, OOL) stability measurements. This second lower-layer (OOL) interferometer provides a direct performance assessment of the actively stabilized DLI, since it uses the same beam path as the DUV/XUV beams for pump-probe experiments. In addition, the HeNe intensity is referenced by another photodiode to eliminate intensity-related drift. Figure 3 shows the phase stability of the DLI measured over $\sim 2 \mathrm{~h}$ at a fixed optical delay. The signal from the upper- and lower-layer 
photodiodes was sampled at a $6 \mathrm{kHz}$ rate (well above the relevant acoustic frequencies) and converted into optical delay units by using the HeNe wavelength of $632.8 \mathrm{~nm}$ and the amplitude of both interference signals obtained from a short scan of the delay stage with the PID controller switched off.
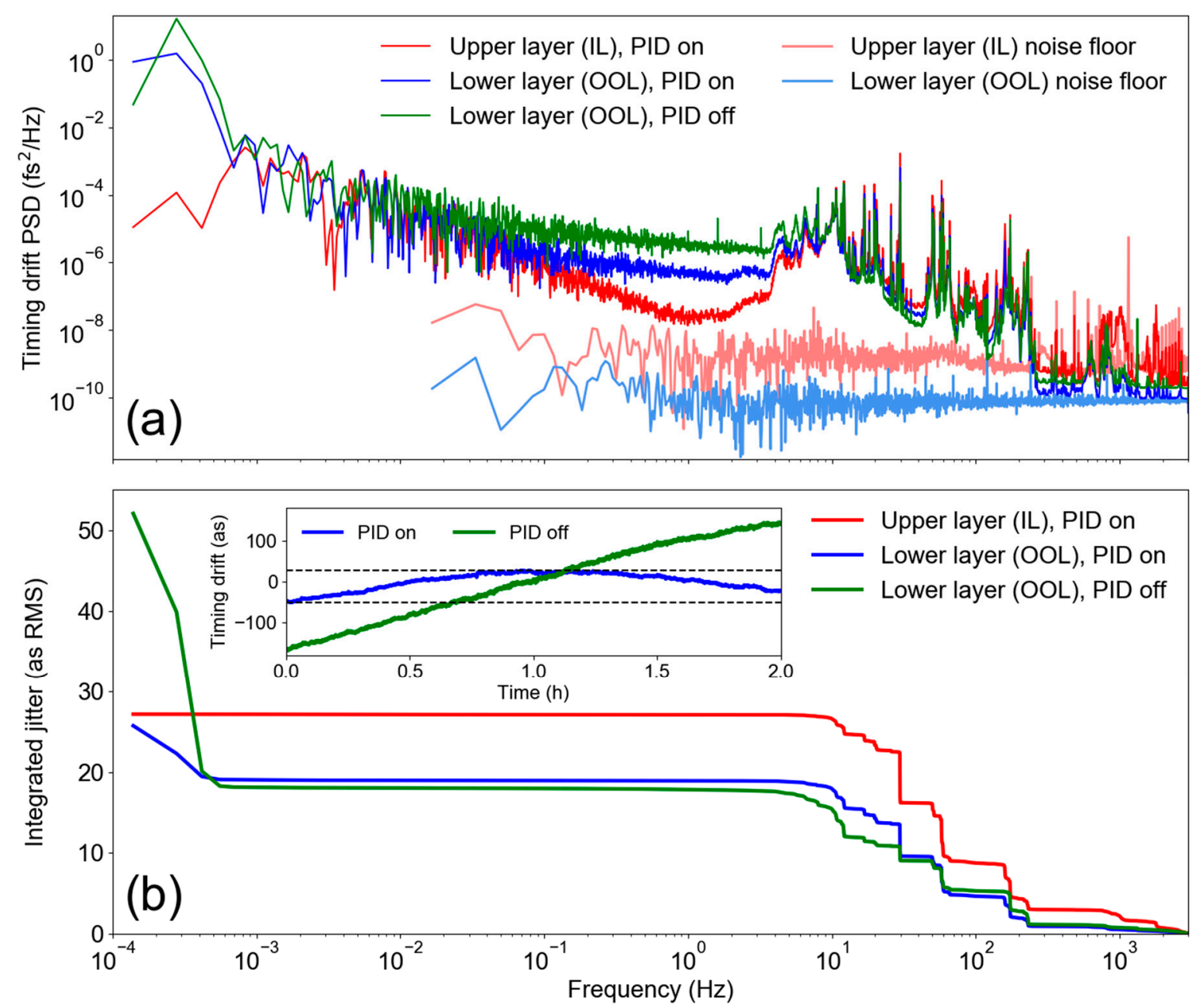

Figure 3. Frequency-domain analysis of the optical delay timing jitter and drift. (a) Power spectral density for the upper layer (IL) (red), lower layer (OOL) with PID control (blue), and lower layer (OOL) without PID control (green) measurements. The instrument noise floor for the respective interferometers is shown in lighter colors. (b) Integrated timing jitter calculated from the PSD shown in (a). Temperature-related long-term drift is notable below $\sim 0.4 \mathrm{mHz}$. The inset shows the time-domain measurement of the drift, which is significantly reduced by the active stabilization on longer time scales.

The power spectral density (PSD) of the timing drift signal of the upper- (IL) and lower-layer (OOL) interferometer as well as their corresponding integrated jitter (squareroot of the integrated PSD, starting at high frequencies) are shown in Figure 3. In order to determine long-term stability, both the upper and lower layer stability were measured for two hours at a fixed optical delay position with and without active PID control. The analog PID controller was not bandwidth limited within the depicted frequency range. In Figure 3, upper layer measurements are shown in red, lower layer in blue, and free-running (PID control disabled) in green. It is evident from Figure 3 a that the active stabilization notably reduces the timing drift in the lower-layer (OOL) interferometer over the entire frequency range below $\sim 15 \mathrm{~Hz}$. On the other hand, the active PID control introduces some noise on its own at higher frequencies due to a mechanical resonance of the piezoelectric stage around $30 \mathrm{~Hz}$ as well as electronic noise, which increases the overall lower-layer (OOL) timing jitter by about three as above $30 \mathrm{~Hz}$ (Figure 3b). Note that the upper layer (IL) measurement had a lower signal-to-noise ratio compared to the lower-layer signal due to a different amount 
of optical power on the respective photodiodes, effecting a higher noise floor and thus a higher apparent timing jitter in the upper layer measurement due to electronic noise. However, only the lower layer measurement provides the relevant stability assessment and upper limit for timing drift in pump-probe experiments, since it uses the DUV / XUV beam path. It is also worth emphasizing that the free-running measurement (PID control disabled) exhibits excellent passive stability across the entire frequency range due to careful vibrational decoupling of the delay chamber from the environment, maintaining a sub-20 as RMS timing jitter down to $\sim 0.4 \mathrm{mHz}(\sim 40 \mathrm{~min})$. This is why active PID control only slightly improves the drift and jitter within this frequency range. The PID controller feedback loop operates close to its sensitivity limit as evidenced by its low-frequency behavior $(<1 \mathrm{~Hz})$, where the residual in-loop timing error exhibits a similar behavior as the natural (free-running) drift PSD (though it has only a negligible contribution to the integrated jitter). Temperature fluctuations in the laboratory on longer time scales lead to a total peak-to-peak timing drift of about 310 as over the $2 \mathrm{~h}$ measurement time without active PID control (see inset in Figure 3b). In contrast, the active stabilization reduces this long-term drift significantly to $\sim 80$ as peak-to-peak. This residual lower-layer (OOL) drift, despite of active PID control, is due to differential mechanical drift of the two interferometer layers with temperature. A more rigid mount construction, e.g., a twin mirror mount from a single piece of metal, as well as better temperature control in the laboratory and in particular within the delay chamber could readily improve this temperature-related drift. In addition, an electronic low-pass filter at the output of the PID controller can be used to ensure the excellent passive performance above $\sim 15 \mathrm{~Hz}$ while reducing the timing drift at lower frequencies. This measurement demonstrates that the passive stability of our DLI is already sufficient to perform short-time (less than $40 \mathrm{~min}$ ) measurements with sub-20 as RMS accuracy without using the PID controller. Active stabilization enables longer measurements over hours and potentially improves short-term stability if electronic high-frequency noise from the PID controller system is filtered out.

The main purpose of the DLI is to provide a phase-locked continuous delay scan while maintaining attosecond timing precision. Figure 4 demonstrates a phase-locked continuous scan over the full optical delay range of $200 \mathrm{fs}$. The optical delay was scanned in $\sim 80$ as steps by rotating the polarizer while maintaining active stabilization. At each delay position, the lower-layer (OOL) interference signal was sampled at a $\sim 83 \mathrm{kHz}$ rate for $2 \mathrm{~s}$. The averaged lower-layer signal is shown in Figure $4 \mathrm{a}$ with the interference fringes normalized to the $[-1,1]$ interval after fitting a sine function to the data. The fit yields a wavelength of $634.8 \mathrm{~nm}$, which is within $0.3 \%$ of the actual $632.8 \mathrm{~nm}$ HeNe wavelength. After normalizing the sampled lower-layer signal, the signal spread at each delay step can be easily converted into optical delay jitter by applying the inverse sine function and calculating the standard deviation, which is depicted in Figure $4 \mathrm{~b}$. Data points within 10\% of the extrema have been removed, since the normalized signal may exceed the $[-1,1]$ interval there due to noise and the inverse sine function would be undefined for these points, skewing the overall jitter statistics. We obtain an average timing jitter of $12.9 \pm 0.8$ as RMS over the entire delay range, which quantifies short-term timing jitter within a frequency range of $0.5 \mathrm{~Hz}(2 \mathrm{~s})$ to the Nyquist frequency of $\sim 41.5 \mathrm{kHz}$. Note that this result is somewhat better than the lower-layer (OOL) timing jitter shown in Figure 3 because it was measured on a different day with a slightly different alignment of the DLI and different signal strength on the photodiodes. This suggests that electronic noise contributes significantly to the apparent timing jitter at high frequencies. Long-term drift as well as phase nonlinearity due to imperfect alignment of the polarization optics manifest as a deviation of the measured interference pattern from an ideal sine curve, which amounts to about $2.4 \%$ RMS in this measurement. Overall, this result not only shows the phaselocked delay scanning capability of our DLI, but also provides sufficiently low timing jitter during the delay scan to perform highly accurate attosecond pump-probe experiments with ultrashort DUV and XUV pulses. 

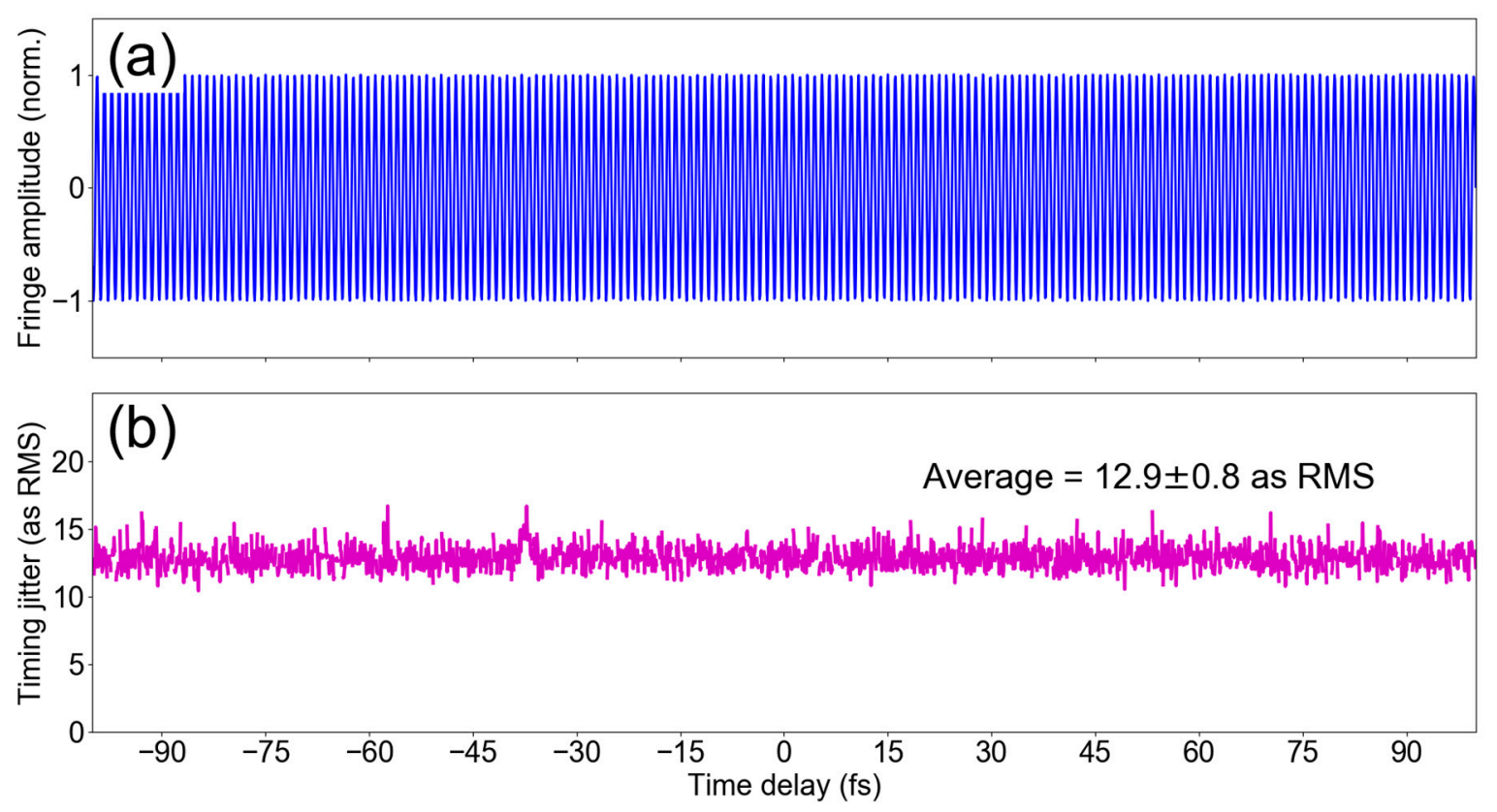

Figure 4. Phase-locked delay scan and stability of the double-layer interferometer over an optical delay range of $200 \mathrm{fs}$. (a) Normalized fringe amplitude in the lower-layer interferometer as a function of optical delay. (b) Timing jitter calculated from the fluctuations in the recorded interference signal within $2 \mathrm{~s}$ of measurement time for each delay step, yielding an average of 12.9 as RMS. Data points within $10 \%$ of the interference extrema have been removed due to unreliable conversion between amplitude and timing there (see text).

\section{Conclusions}

In summary, we implemented a collinear scheme to generate few-cycle DUV and XUV pulses in a gas, separating the resulting pulses spectrally and spatially by a perforated dichroic beam separator, forming a pump-probe experimental configuration. Additionally, we have demonstrated that by utilizing the Pancharatnam's phase stabilization scheme in a DLI, the timing jitter between the pump and probe pulses can be stabilized down to $12.9 \pm 0.8$ as RMS of short-term jitter $(0.5 \mathrm{~Hz}-41.5 \mathrm{kHz})$ and $\sim 80$ for long-term peakto-peak drift over $\sim 2 \mathrm{~h}$. This allows continuous pump-probe delay scans over a range of $200 \mathrm{fs}$ with attosecond timing precision. In addition, we demonstrated exceptional sub-20 as passive stability of our system, only requiring active stabilization to compensate for long-term thermal drift on $>40 \mathrm{~min}$ time scales. A DLI setup solves the challenge of using a visible-light reference laser for optical phase stabilization and phase-locked delay scanning with a DUV and XUV pump-probe configuration. This excellent optical phase stability, combined with the ultrashort DUV/XUV pulses, enables the investigation of femtosecond and attosecond electron dynamics [28] with the highest possible timing precision, which is faster than the molecule's vibrational response.

Author Contributions: Conceptualization, D.-E.K.; methodology, T.K. and A.G.; software, A.G.; validation, T.K., A.G. and J.H.; formal analysis, T.K., A.G. and J.-H.M. investigation, T.K. and J.-H.M.; resources, Y.L.; data curation, T.K. and A.G.; writing-original draft preparation, T.K. and A.G.; writing-review and editing, T.K., A.G. and D.-E.K.; visualization, T.K. and Y.L.; supervision, D.-E.K.; project administration, D.-E.K.; funding acquisition, D.-E.K. All authors have read and agreed to the published version of the manuscript.

Funding: This research was funded by the Max Planck POSTECH/KOREA Research Initiative Program, grant number 2016K1A4A4A01922028, through the National Research Foundation of Korea (NRF) funded by the Ministry of Science, ICT \& Future Planning, and in part by the National Research Foundation of Korea (NRF), grant numbers 2020R1C1C1012953 and 2021R1I1A1A01057547.

Institutional Review Board Statement: Not applicable. 
Informed Consent Statement: Not applicable.

Data Availability Statement: The data presented in this study are available on request from the corresponding author. The data are not publicly available due to ethical restrictions.

Conflicts of Interest: The authors declare no conflict of interest.

\section{References}

1. Fleming, G.R.; Ratner, M.A. Grand challenges in basic energy sciences. Phys. Today 2008, 61, 28-33. [CrossRef]

2. Martin, F.; Hishikawa, A.; Vrakking, M. Special issue on ultrafast electron and molecular dynamics. J. Phys. B At. Mol. Opt. Phys. 2014, 47. [CrossRef]

3. Nisoli, M.; Sansone, G. New frontiers in attosecond science. Prog. Quantum Electron. 2009, 33, 17-59. [CrossRef]

4. Sansone, G.; Pfeifer, T.; Simeonidis, K.; Kuleff, A.I. Electron correlation in real time. ChemPhysChem 2012, 13, 661-680. [CrossRef]

5. Mignolet, B.; Levine, R.D.; Remacle, F. Localized electron dynamics in attosecond-pulse-excited molecular systems: Probing the time-dependent electron density by sudden photoionization. Phys. Rev. A-At. Mol. Opt. Phys. 2012, 86, 1-8. [CrossRef]

6. Kuleff, A.I.; Cederbaum, L.S. Ultrafast correlation-driven electron dynamics. J. Phys. B At. Mol. Opt. Phys. 2014, 47. [CrossRef]

7. Nest, M.; Remacle, F.; Levine, R.D. Pump and probe ultrafast electron dynamics in LiH: A computational study. New J. Phys. 2008, 10. [CrossRef]

8. Chang, B.Y.; Shin, S.; Palacios, A.; Martín, F.; Sola, I.R. Oscillating molecular dipoles require strongly correlated electronic and nuclear motion. J. Phys. B At. Mol. Opt. Phys. 2015, 48. [CrossRef]

9. Remacle, F.; Levine, R.D. An electronic time scale in chemistry. Proc. Natl. Acad. Sci. USA 2006, 103, 6793-6798. [CrossRef]

10. Braun, H.; Bayer, T.; Sarpe, C.; Siemering, R.; De Vivie-Riedle, R.; Baumert, T.; Wollenhaupt, M. Coupled electron-nuclear wavepacket dynamics in potassium dimers. J. Phys. B At. Mol. Opt. Phys. 2014, 47. [CrossRef]

11. Wörner, H.J.; Arrell, C.A.; Banerji, N.; Cannizzo, A.; Chergui, M.; Das, A.K.; Hamm, P.; Keller, U.; Kraus, P.M.; Liberatore, E.; et al. Charge migration and charge transfer in molecular systems. Struct. Dyn. 2017, 4, 061508. [CrossRef]

12. Calegari, F.; Trabattoni, A.; Palacios, A.; Ayuso, D.; Castrovilli, M.C.; Greenwood, J.B.; Decleva, P.; Martín, F.; Nisoli, M. Charge migration induced by attosecond pulses in bio-relevant molecules. J. Phys. B At. Mol. Opt. Phys. 2016, 49. [CrossRef]

13. Mignolet, B.; Levine, R.D.; Remacle, F. Charge migration in the bifunctional PENNA cation induced and probed by ultrafast ionization: A dynamical study. J. Phys. B At. Mol. Opt. Phys. 2014, 47. [CrossRef]

14. Graf, U.; Fieß, M.; Schultze, M.; Kienberger, R.; Krausz, F.; Goulielmakis, E. Intense few-cycle light pulses in the deep ultraviolet. Opt. Express 2008, 16, 18956. [CrossRef]

15. Reiter, F.; Graf, U.; Serebryannikov, E.E.; Schweinberger, W.; Fiess, M.; Schultze, M.; Azzeer, A.M.; Kienberger, R.; Krausz, F.; Zheltikov, A.M.; et al. Route to attosecond nonlinear spectroscopy. Phys. Rev. Lett. 2010, 105, 1-4. [CrossRef] [PubMed]

16. Galli, M.; Wanie, V.; Lopes, D.P.; Månsson, E.P.; Trabattoni, A.; Colaizzi, L.; Saraswathula, K.; Cartella, A.; Frassetto, F.; Poletto, L.; et al. Generation of deep ultraviolet sub-2-fs pulses. Opt. Lett. 2019, 44, 1308. [CrossRef]

17. Paul, P.M.; Toma, E.S.; Breger, P.; Mullot, G.; Augé, F.; Balcou, P.; Muller, H.G.; Agostini, P. Observation of a train of attosecond pulse from high harmonic generation. Science 2001, 292, 1689-1692. [CrossRef] [PubMed]

18. Sun, H.-W.; Huang, P.-C.; Tzeng, Y.-H.; Huang, J.-T.; Lin, C.D.; Jin, C.; Chen, M.-C. Extended phase matching of high harmonic generation by plasma-induced defocusing. Optica 2017, 4, 976. [CrossRef]

19. Teichmann, S.M.; Silva, F.; Cousin, S.L.; Hemmer, M.; Biegert, J. 0.5-keV Soft X-ray attosecond continua. Nat. Commun. 2016, 7, 1-6. [CrossRef]

20. Chini, M.; Mashiko, H.; Wang, H.; Chen, S.; Yun, C.; Scott, S.; Gilbertson, S.; Chang, Z. Delay control in attosecond pump-probe experiments. Opt. Express 2009, 17, 21459. [CrossRef]

21. Sabbar, M.; Heuser, S.; Boge, R.; Lucchini, M.; Gallmann, L.; Cirelli, C.; Keller, U. Combining attosecond XUV pulses with coincidence spectroscopy. Rev. Sci. Instrum. 2014, 85. [CrossRef] [PubMed]

22. Schlaepfer, F.; Volkov, M.; Hartmann, N.; Niedermayr, A.; Schumacher, Z.; Gallmann, L.; Keller, U. Phase stabilization of an attosecond beamline combining two IR colors. Opt. Express 2019, 27, 22385. [CrossRef] [PubMed]

23. Silva, F.; Miranda, M.; Alonso, B.; Rauschenberger, J.; Pervak, V.; Crespo, H. Simultaneous compression, characterization and phase stabilization of GW-level 14 cycle VIS-NIR femtosecond pulses using a single dispersion-scan setup. Opt. Express 2014, 22, 10181. [CrossRef]

24. Bothschafter, E.M.; Schiffrin, A.; Yakovlev, V.S.; Azzeer, A.M.; Krausz, F.; Ernstorfer, R.; Kienberger, R. Collinear generation of ultrashort UV and XUV pulses. Opt. Express 2010, 18, 9173. [CrossRef] [PubMed]

25. Schötz, J.; Förg, B.; Schweinberger, W.; Liontos, I.; Masood, H.A.; Kamal, A.M.; Jakubeit, C.; Kling, N.G.; Paasch-Colberg, T.; Biswas, S.; et al. Phase-Matching for Generation of Isolated Attosecond XUV and Soft-X-Ray Pulses with Few-Cycle Drivers. Phys. Rev. X 2020, 10, 41011. [CrossRef]

26. Kling, N.G.; Paul, D.; Gura, A.; Laurent, G.; De, S.; Li, H.; Wang, Z.; Ahn, B.; Kim, C.H.; Kim, T.K.; et al. Thick-lens velocity-map imaging spectrometer with high resolution for high-energy charged particles. J. Instrum. 2014, 9, P05005. [CrossRef] 
27. Wehner, M.U.; Ulm, M.H.; Wegener, M. Scanning interferometer stabilized by use of Pancharatnam's phase. Opt. Lett. 1997, 22, 1455. [CrossRef] [PubMed]

28. Nisoli, M.; Decleva, P.; Calegari, F.; Palacios, A.; Martín, F. Attosecond electron dynamics in molecules. Chem. Rev. 2017, 117, 10760-10825. [CrossRef] [PubMed] 